\title{
Aspectos determinantes do posicionamento corporal no ciclismo: uma revisão sistemática
}

\author{
Julio Francisco Kleinpaul ${ }^{1}$ \\ Luana Mann ${ }^{1}$ \\ Fernando Diefenthaeler ${ }^{1}$ \\ Antônio Renato Pereira Moro ${ }^{1}$ \\ Felipe Pivetta Carpes ${ }^{2}$ \\ ${ }^{1}$ Centro de Desportos, Universidade Federal de Santa Catarina, Florianópolis, SC, Brasil \\ ${ }^{2}$ Centro de Ciências da Saúde, Universidade Federal do Pampa, Uruguaiana, RS, Brasil
}

\begin{abstract}
Resumo: Através de revisão sistemática, fez-se um levantamento de estudos que tratam do posicionamento corporal adequado para o ciclismo. Para isso, buscou-se por referências em língua Portuguesa e Inglesa, nas bases de dados LILACS, ScienceDirect, SciELO e MEDLINE. Os descritores utilizados para a busca dos artigos foram: posicionamento corporal + ciclismo; ajuste + bicicleta; postura + ciclismo; body positioning + cycling; bicycle fitting; cycling posture. Foram considerados artigos originais, de revisão simples, dissertações de mestrado e livros que tratassem de forma clara e objetiva o assunto, desde que publicados entre 1993 e 2009 (outubro). No total 20 estudos atenderam esses requisitos. De modo geral, estes sugerem que o conhecimento e habilidade para ajustar a bicicleta são úteis, no entanto, mesmo existindo protocolos para o ajuste adequado da bicicleta ao ciclista, os estudos denotam que a maioria dos ciclistas ainda as utiliza de forma errônea. Isso sugere a dificuldade de acesso aos estudos.
\end{abstract}

Palavras-chave: Bicicleta. Desempenho. Ergonomia.

\section{Aspects determinants of body positioning for cycling: a systematic review}

\begin{abstract}
By means of systematic review a surveying of English and Portuguese language studies concerning the expected body positioning for improvement of cycling. Search engines used were LILACS, ScienceDirect, SciELO and MEDLINE. The keywords used to find relevant papers were: posicionamento corporal + ciclismo; ajuste + bicicleta; postura + ciclismo; body positioning + cycling; bicycle fitting; cycling posture. Original papers, short review papers, master thesis and books published from 1993 to 2009 (October) were considered when the main subject of discussion was the topic of interest. A total of 20 studies were considered. In general, the studies suggest that is will be useful to know about the bicycle fitting. Even so protocols for body positioning evaluation are available, most of studies concluded that cyclists are not able to use this protocol correctly. It suggests the difficult to access studies.
\end{abstract}

Key Words: Bicycle. Performance. Ergonomics.

\section{Introdução}

Exercícios de baixa intensidade e longa duração são os mais indicados para pessoas que buscam melhorar o condicionamento físico e a melhora da qualidade de vida. Isso faz com que o número de adeptos a esportes como o ciclismo, seja ele indoor (spinning, por exemplo) ou na rua, aumente a cada ano. Isso faz com que o ciclismo seja um dos esportes mais tradicionais no mundo, tanto em relação a número de ciclistas e triatletas profissionais como praticantes em geral. Além do caráter competitivo, o ciclismo tem se tornado uma forma de lazer bem como um excelente meio de locomoção (ROWE, HULL e WANG, 1998; BURKE, 2000; CARMO, 2001).

De acordo com a Agência Nacional de Transportes Terrestres (ANTT), a bicicleta é diariamente usada por cerca de quatro milhões de pessoas. Como exemplo, uma comparação entre os anos de 1997 e 2008 revela um aumento de $189 \%$ no número de bicicletas circulando nas ruas brasileiras (ABRACICLO, 2008). Uma preocupação que surge com isso é a saúde dos usuários, não somente em relação ao trânsito, mas também a ergonomia. Os aspectos relacionados à ergonomia, nesse caso, dizem respeito ao ajuste correto da interação ciclistabicicleta na busca tanto por conforto como por desempenho e bem-estar. Diferentes métodos e padrões de ajustes na regulagem desse complexo têm sido propostos, na busca tanto por conforto como por desempenho do ciclista. Existem inúmeras discussões acerca de como o ciclista deve se posicionar na bicicleta, isto é, qual o posicionamento ideal para maximização do conforto e do desempenho. Um dos grandes causadores do abandono por parte dos 
praticantes iniciantes são as dores no corpo provocadas muitas vezes pela má postura e por desajustes nos equipamentos utilizados como, por exemplo, o ajuste da posição do selim (BURKE, 1996; DIEFENTHAELER et al., 2008b).

Seja com a finalidade de competir ou simplesmente por lazer, a bicicleta deve possuir os ajustes na medida correta para o objetivo pretendido. Essas regulagens não devem somente se ater ao tamanho do quadro, que é a principal medida na escolha da bicicleta, mas também ao posicionamento do selim em relação à posição horizontal e vertical (DIEFENTHAELER et al., 2008b), posição do guidom e tamanho do pé-de-vela, partes essas que geralmente formam as partes móveis da bicicleta. Esses ajustes merecem especial atenção, pois estas partes móveis podem ser reguladas de acordo com a dimensão corporal do ciclista. A geometria do complexo ciclista-bicicleta pode influenciar a magnitude e a direção de aplicação das forças no pedal, técnica da pedalada, estratégia neuromuscular adotada, economia de movimento, probabilidade de lesões, atrito aerodinâmico e, mais diretamente, sensação de conforto na bicicleta (WELBERGEN e CLIJSEN, 1990; MESTDAGH, 1998). Pequenas modificações nos ajustes do complexo ciclista-bicicleta podem afetar as propriedades mecânicas (por exemplo, a relação força-comprimento e força-velocidade) e elétricas dos músculos envolvidos no movimento da pedalada (HERZOG et al., 1991; DIEFENTHAELER et al., 2008b).

Dessa forma, ao observarmos o dia-a-dia dos ciclistas vemos que suas posturas, geralmente, não estão totalmente corretas. Este posicionamento incorreto está, na maioria das vezes, relacionado à falta de informação que os ciclistas têm sobre a maneira adequada de adaptar a bicicleta às dimensões do seu corpo (MARTINS et al., 2007). Considerando as medidas quantitativas de regulagem da posição disponíveis na literatura específica (livros e revistas especializadas), percebe-se que muitos ciclistas adotam ajustes baseados apenas em sensações subjetivas (MESTDAGH, 1998; DIEFENTHAELER et al., 2008a; DIEFENTHAELER et al., 2008b). Geralmente os ciclistas se adaptam a essas regulagens ao longo dos anos. No entanto, esses ajustes talvez não sejam os mais apropriados em termos de aproveitamento das forças aplicadas no pedal e economia de movimento, havendo assim a necessidade de métodos mais específicos que considerem questões individuais na avaliação do posicionamento (DIEFENTHAELER et al., 2008b). Além das perdas na técnica e na eficiência, ciclistas são regularmente confrontados com lesões e dores crônicas causadas por desvios na posição ou posições inadequadas na bicicleta (BURKE, 1996), o que tem sido efetivamente minimizado quando o posicionamento é corrigido (WELBERGEN e CLIJSEN, 1990; BURKE, 1996).

Até o presente, nenhuma revisão sistemática foi conduzida com a finalidade de analisar os estudos que tratem dos ajustes no posicionamento adequado ao ciclismo, o que se torna uma questão crítica quando considerando o número de praticantes dessa modalidade. Com o intuito de apresentar os aspectos atuais que vêm sendo considerados por pesquisadores da área, e mostrar quais as estratégias que mais vêm apresentando sucesso, o objetivo deste estudo foi analisar as pesquisas realizadas considerando medidas determinantes do posicionamento corporal e os fatores que o ajuste corporal pode afetar em relação à prática esportiva e uso da bicicleta como meio de transporte.

\section{Métodos}

Para o desenvolvimento do presente estudo foram considerados artigos originais, artigos de revisão, dissertações de mestrado e livros com menção ao posicionamento na bicicleta, que tratassem de forma clara e objetiva 0 assunto. Para pesquisa, foram consideradas quatro bases de dados eletrônicas: LILACS ${ }^{\circledR}$ (Literatura LatinoAmericana e do Caribe em Ciências da Saúde), ScienceDirect ${ }^{\Theta}$ (Elsevier), SciELO ${ }^{\circledR}$ (Scientific Electronic Library Online) e MEDLINE ${ }^{\circledR}$, e suas publicações entre o período de janeiro de 1993 e outubro de 2009 disponíveis em versão final. A seleção dos descritores utilizados no processo de revisão foi efetuada mediante consulta aos DECs (descritores de assunto em ciências da saúde da BIREME). Nas buscas, os seguintes descritores, em língua Portuguesa e Inglesa, foram considerados: posicionamento corporal + ciclismo; ajuste + bicicleta; postura + ciclismo; body positioning + cycling; bicycle fitting; cycling posture. Recorreu-se aos operadores boolenos "AND" e "OR" para combinação dos descritores e termos utilizados para rastreamento das publicações.

A busca nas bases eletrônicas de dados foi realizada no mês de outubro de 2009. Após a primeira análise, com avaliação dos títulos, 38 artigos foram considerados elegíveis para a segunda fase desta revisão, que consistiu na leitura dos resumos. Após avaliação dos resumos quanto à relevância ao objetivo deste estudo, as referências que preenchiam os critérios de inclusão foram lidas na íntegra. Ao final, 20 estudos atenderam a todos os critérios para 
inclusão. Foram incluídos, ainda, outros 10 estudos que haviam sido citados na lista de referências dos 20 manuscritos, inicialmente incluídos no estudo, totalizando então 30 estudos.

Para a avaliação dos artigos, observaram-se os seguintes aspectos:

- tipo de ajuste (geral ou específico);

- amostra ou grupo de estudo (dimensionamento, indivíduos avaliados);

- principais resultados.

\section{Resultados}

Quanto as informações gerais dos 20 estudos incluídos, 16 foram publicados no período de 2000 a 2009. Apenas três estudos são de revisão (MESTDAGH, 1998; SILBERMAN et al., 2005; ALENCAR e MATIAS, 2009), um livro (BURKE e PRUITT, 2003) e uma Dissertação de Mestrado (PEQUINI, 2000). A composição das amostras não variou muito quanto à faixa etária, sendo que todos envolveram adultos jovens (exceto os estudo de Donkers et al. (1993) e Laios e Giannatsis (2009) que envolveram crianças) e, dos artigos que se referem ao sexo da amostra, sete deles incluíram mulheres (CHRISTIAANS e BREMNER, 1998; SALAl et al., 1999; BRESSEL $\mathrm{e}$ LARSON, 2003; SAVELBERG, VAN de PORT e WILLEMS, 2003; PEVELER et al., 2005; PEVELER, POUNDERS e BISHOP, 2007; SAUER et al., 2007).

Destes estudos, quatro foram desenvolvidos no Brasil (PEQUINI, 2000; MARTINS et al., 2007; DIEFENTHAELER et al., 2008a; ALENCAR e MATIAS, 2009). Em se tratando de estudos de levantamento e descrição de características de uma população, torna-se importante avaliar um número grande de sujeitos. No entanto, apenas seis estudos incluíram amostra superior a 30 indivíduos (CHRISTIAANS e BREMNER, 1998; SALAI et al., 1999; PEQUINI, 2000; MARTINS et al., 2007; DONKERS et al., 1993; LAIOS e GIANNATSIS, 2009).

Na Tabela 1, estão apresentados os tipos de ajustes utilizados (geral quando considerado os ajustes da bicicleta como um todo, e específico quando considerado apenas um ajuste), e o público para o qual foi dirigido o trabalho. Destes, 10 estudos utilizaram ajustes gerais da bicicleta para o melhor posicionamento dos ciclistas (DONKERS et al., 1993; CHRISTIAANS e BREMNER, 1998; MESTDAGH, 1998; PEQUINI, 2000; BURKE e PRUITT, 2003; SILBERMAN et al., 2005; MARTINS et al., 2007; SAUER et al., 2007; ALENCAR e MATIAS, 2009; LAIOS e GIANNATSIS, 2009). Dentre os demais, quatro referiram-se apenas ao ajuste do selim (SALAl et al., 1999; PEVELER et al., 2005; PEVELER,
POUNDERS e BISHOP, 2007; DIEFENTHAELER et al., 2008a), cinco ao posicionamento do tronco do ciclista sobre a bicicleta (GRAPPE et al., 1998; ASHE et al., 2003; SAVELBERG, VAN de PORT e WILLEMS, 2003; DOREL, COUTURIER e HUG, 2007; DUC et al., 2008) e um ao posicionamento do tronco e o tipo de selim (BRESSEL e LARSON, 2003). Os ciclistas competitivos foram alvo de sete estudos (GRAPPE et al., 1998; PEQUINI, 2000; PEVELER et al., 2005; DOREL, COUTURIER e HUG, 2007; SAUER et al., 2007; DIEFENTHAELER et al., 2008a; DUC et al., 2008), e em outros seis foram os ciclistas recreacionais (DONKERS et al., 1993; CHRISTIAANS e BREMNER, 1998; SALAI et al., 1999; ASHE et al., 2003; SAVELBERG, VAN de PORT e WILLEMS, 2003; LAIOS e GIANNATSIS, 2009). Os sete restantes estudaram ambos (MESTDAGH, 1998; BRESSEL e LARSON, 2003; BURKE e PRUITT, 2003; SILBERMAN et al., 2005; MARTINS et al., 2007; PEVELER, POUNDERS e BISHOP, 2007; ALENCAR e MATIAS, 2009).

Dentre os estudos que consideraram os efeitos do posicionamento (ou de ajustes/correções) sobre aspectos fisiológicos dos ciclistas, quatro consideraram o consumo de oxigênio (GRAPPE et al., 1998; ASHE et al., 2003; DOREL, COUTURIER e HUG, 2007; DIEFENTHAELER et al., 2008a), dois a produção de dióxido de carbono (DOREL, COUTURIER e HUG, 2007; DIEFENTHAELER et al., 2008a), e um a freqüência cardíaca (ASHE et al., 2003). Aspectos biomecânicos como a cinemática foram o foco central de cinco estudos (SALAl et al., 1999; SAVELBERG, VAN de PORT e WILLEMS, 2003; SILBERMAN et al., 2005; SAUER et al., 2007; DIEFENTHAELER et al., 2008a), cinética de outros quatro estudos (ASHE et al., 2003; DOREL, COUTURIER e HUG, 2007; PEVELER, POUNDERS e BISHOP, 2007; SAUER et al., 2007) e eletromiografia em quatro estudos (SAVELBERG, VAN de PORT e WILLEMS, 2003; DOREL, COUTURIER O HUG, 2007; DIEFENTHAELER et al., 2008a; DUC et al., 2008). O número de estudos que considerou fatores fisiológicos e biomecânicos conjuntos foi de três (ASHE et al., 2003; DOREL, COUTURIER e HUG, 2007; DIEFENTHAELER et al., 2008a). Dentre os estudos, nove referiram-se explicitamente a ajustes antropométricos aplicados no dimensionamento da bicicleta (DONKERS et al., 1993; CHRISTIAANS e BREMNER, 1998; MESTDAGH, 1998; PEQUINI, 2000; SILBERMAN et al., 2005; DOREL, COUTURIER e HUG, 2007; SAUER et al., 2007; ALENCAR e MATIAS, 2009; LAIOS e GIANNATSIS, 2009). 
Tabela 1. Análise dos artigos selecionados de acordo com os ajustes utilizados e o nível de treinamento dos sujeitos. Os artigos estão listados em ordem cronológica, considerando o nome do primeiro autor, ano da publicação, os ajustes realizados ou recomendados e o público do qual a amostra foi composta (público alvo).

\begin{tabular}{|c|c|c|c|c|}
\hline Referência & $\begin{array}{c}\text { Idade } \\
\text { (média } \pm \text { DP em } \\
\text { anos) }\end{array}$ & $\mathrm{N}$ & Ajustes & Público alvo \\
\hline Laios e Giannatsis (2009) & $7-14$ & 1247 & Gerais & Recreacional \\
\hline Alencar e Matias (2009) & --- & --- & Gerais & Recreacional e Competitivo \\
\hline Diefenthaeler (2008a) & $26 \pm 4$ & 3 & Altura e avanço do selim & Competitivo \\
\hline$\underline{\text { Duc et al. }(2008)}$ & $28 \pm 7$ & 10 & Posicionamento do tronco & Competitivo \\
\hline Dorel (2007) & $31 \pm 8$ & 12 & Posicionamento do tronco & Competitivo \\
\hline Martins (2007) & $28 \pm 10$ & 36 & Gerais & Recreacional e Competitivo \\
\hline$\underline{\text { Peveler (2007) }}$ & $25 \pm 3$ & 27 & Altura do selim & Recreacional e Competitivo \\
\hline$\underline{\text { Sauer (2007) }}$ & $36 \pm 11$ & 26 & Gerais & Competitivo \\
\hline Peveler (2005) & $29 \pm 6$ & 19 & Altura do selim & Competitivo \\
\hline$\underline{\text { Silberman (2005) }}$ & --- & --- & Gerais & Recreacional e Competitivo \\
\hline Ashe (2003) & $19 \pm 1$ & 12 & Posicionamento do tronco & Recreacional \\
\hline Bressel e Larson (2003) & $24 \pm 6$ & 20 & $\begin{array}{l}\text { Posicionamento do tronco e } \\
\text { tipo de selim }\end{array}$ & Recreacional e Competitivo \\
\hline Burke (2003) & --- & --- & Gerais & Recreacional e Competitivo \\
\hline$\underline{\text { Savelberg (2003) }}$ & $22 \pm 1$ & 8 & Posicionamento do tronco & Recreacional \\
\hline Pequini $(2000)$ & --- & 40 & Gerais & Competitivo \\
\hline$\underline{\text { Salai (1999) }}$ & $17-72$ & 40 & Inclinação do selim & Recreacional \\
\hline$\underline{\text { Christiaans (1998) }}$ & $46 \pm 13$ & 132 & Gerais & Recreacional \\
\hline Grappe et al. (1998) & $26 \pm 3$ & 9 & Posicionamento do tronco & Competitivo \\
\hline Mestdagh (1998) & --- & --- & Gerais & Recreacional e Competitivo \\
\hline Donkers (1993) & $2,5-5,5$ & 279 & Gerais & Recreacional \\
\hline
\end{tabular}

Em 13 dos 20 estudos, o ajuste da bicicleta fez parte do objetivo principal (DONKERS et al., 1993; CHRISTIAANS e BREMNER, 1998; MESTDAGH, 1998; SALAI et al., 1999; PEQUINI, 2000; BRESSEL e LARSON, 2003; BURKE e PRUITT, 2003; PEVELER et al., 2005; SILBERMAN et al., 2005; MARTINS et al., 2007; DIEFENTHAELER et al., 2008a; ALENCAR e MATIAS, 2009; LAIOS e GIANNATSIS, 2009), enquanto que nos demais o ajuste serviu apenas para padronizar e equiparar os sujeitos (manter sempre a mesma posição dentre diferentes coletas) durante as avaliações (GRAPPE et al., 1998; ASHE et al., 2003; SAVELBERG, VAN de PORT \& WILLEMS, 2003; DOREL, COUTURIER e HUG, 2007; PEVELER, POUNDERS e BISHOP. 2007; SAUER et al., 2007; DUC et al., 2008).

\section{Discussão}

Muitas vezes o ajuste da bicicleta ainda é feito numa base de tentativa e erro. Ajustes incorretos no posicionamento podem levar à lesão, pois podem acarretar aumento da sobrecarga músculo-esquelética (BURKE, 1996). O pedalar não é um movimento natural na ergonomia do ser humano, e como conseqüência disso, a menor irregularidade no campo da simetria física pode levar a todo tipo de reclamações (MARTINS et al., 2007). No ciclismo, a ergonomia tem como objetivo principal aumentar a segurança, a satisfação e o bem-estar dos ciclistas, isto é, procura adaptar a bicicleta à estrutura humana para que se alcance $o$ melhor rendimento na pedalada (PEQUINI, 2000).

A pesquisa científica e empírica tem mostrado que a posição correta numa bicicleta é determinada por vários fatores. O sistema que ilustra a correlação entre eles é formado pelos seguintes fatores: fricção, eficiência, maximização da potência da pedalada e conforto. Contudo, aparentemente, muitos ciclistas amadores, e até mesmo profissionais, continuam a adotar posições incorretas nas suas bicicletas (BURKE e PRUITT, 2003; MARTINS et al., 2007).

Em um dos estudos sobre posicionamento com maior riqueza em detalhes de descrição das 
avaliações e variáveis consideradas para o bom posicionamento do ciclista, Martins et al. (2007) avaliaram e compararam o posicionamento na bicicleta adotado por 36 ciclistas de diferentes níveis. Os ciclistas foram avaliados em suas próprias bicicletas e classificados como competitivos (atletas, $\mathrm{n}=19$ ) e recreacionais (não-atletas, $\mathrm{n}=17$ ) com base no histórico de prática. Para as avaliações foi utilizado um protocolo a partir do proposto por Burke e Pruitt (2003). Foram detectados desajustes no posicionamento em $82 \%$ dos ciclistas recreacionais e $74 \%$ dos ciclistas competitivos avaliados. Para os ajustes no selim foram encontrados os erros mais comuns, relacionados com a posição vertical e horizontal do selim (observados para $82 \%$ dos ciclistas recreacionais e $79 \%$ dos ciclistas competitivos). O segundo erro com um grande número de observações foi relativo a altura do guidom (observados em 12\% dos ciclistas recreacionais e $5 \%$ dos ciclistas competitivos). Com base nestes resultados, percebe-se que os ciclistas recreacionais estão mais suscetíveis a desajustes no posicionamento do que ciclistas competitivos, 0 que pode acarretar lesões no futuro. Os autores concluem que os erros no posicionamento encontrados podem estar relacionados ao tempo de prática (experiência).

Mestdagh (1998), Pequini (2000), Burke e Pruitt (2003), Silberman et al. (2005) e Alencar e Matias (2008) fizeram um levantamento geral dos ajustes a serem adotados para selim, guidom, posicionamento dos pés sobre 0 pedal, e tamanho adequado do quadro da bicicleta além de outros fatores que possam contribuir para 0 bom posicionamento do ciclista. A conclusão geral foi de que o ajuste da bicicleta deve ser individualizado para que se alcance 0 melhor desempenho, conforto, satisfação e prevenção de lesões. Mestdagh (1998) ressalta que os ajustes podem ser baseados apenas nas medidas antropométricas externas dos ciclistas, enquanto Silberman et al. (2005) sugerem ainda a utilização de ajustes tanto estáticos quanto dinâmicos. Porém, quando se trata de ciclistas de elite as regulagens do complexo ciclista-bicicleta devem respeitar as características individuais, pois segundo Diefenthaeler et al. (2008b), ajustes relativamente pequenos podem exercer grande influência no desempenho desses atletas.

Seguindo a mesma tendência, Christiaans e Bremner (1998) realizaram estudo detalhado dos ajustes corretos a serem utilizados na regulagem geral de bicicletas. $O$ foco foi a mensuração das preferências adotadas pelos ciclistas e a comparação com medidas corporais. O estudo procurou investigar fatores relacionados com o conforto e a validade dos ajustes relacionados. Os resultados mostraram que os ajustes encontrados nas bicicletas comerciais são questionáveis por não levarem em conta fatores individuais de medidas antropométricas.

Em um projeto intitulado KIMA-1, Donkers et al. (1993) estudaram 33 dimensões de 279 crianças (2,5 - 5,5 anos). Estes dados foram usados para comparar dimensões corporais de crianças com dimensões de bicicletas. Além disso, as exigências relativas às dimensões das bicicletas consideradas seguras, encontradas com o programa KIMA-1, foram comparadas com algumas bicicletas disponíveis no mercado. Os autores concluíram que máxima segurança e conforto da bicicleta são alcançados quando a mesma for provida de dimensões adequadas à criança, concordando com os achados de Laios e Giannatsis (2009). Economias no processo de ajuste podem ser alcançadas relacionando as dimensões da bicicleta à estatura ao invés da idade da criança. Independente disso, mais uma vez, a individualidade do ajuste da bicicleta foi ressaltada como fundamental.

As diferentes posições adotadas pelos atletas em função de diferentes modelos de guidom e selim também são tópicos de muito interesse. Segundo Mestdagh (1998), o ajuste do selim deve ser o primeiro a ser realizado. Ele é o principal apoio para o ciclista, e a sua posição relativa ao movimento central (eixo do pé-de-vela) determinará as condições ergonômicas do movimento das pernas. A seguir vem o guidom, que deve estar colocado de maneira que o ciclista possa pedalar em posição de procura de velocidade (aerodinâmica), e em posição mais elevada para períodos de tempo mais longo, com as mãos repousando na parte superior do guidom, para o caso do ciclismo de estrada. Não é possível corrigir uma postura errada do selim manipulando a posição do guidom, a qual, aliás, pode variar de acordo com as provas. O comprimento do pé-de-vela é relacionado com o comprimento do membro inferior. Este ajuste pode afetar o conforto e a flexão do joelho e quadril. Em caso de mudança no tamanho do péde-vela a altura do selim deverá ser reajustada (MESTDAGH, 1998; BURKE e PRUITT, 2003; ALENCAR E MATIAS, 2009).

Diefenthaeler et al. (2008a) analisaram os efeitos de diferentes posicionamentos do selim no padrão de ativação elétrica durante a pedalada. Três ciclistas de elite foram submetidos a um protocolo que constou da avaliação de quatro diferentes posições de selim (para frente, para trás, para cima e para baixo), assumindo como 
posição de referência a posição usada pelos atletas durante os treinos e competições. O deslocamento do selim foi de $1 \mathrm{~cm}$ para todas as posições. As bicicletas dos ciclistas foram acopladas a um ciclossimulador magnético. A carga do teste foi normalizada utilizando um critério fisiológico, o coeficiente respiratório, garantindo assim o mesmo nível de esforço para todos os sujeitos. A atividade elétrica de seis músculos do membro inferior foi mensurada: glúteo máximo, reto femoral, bíceps femoral, vasto lateral, gastrocnêmio medial e tibial anterior. Os resultados demonstraram que pequenos ajustes na posição do selim podem afetar os padrões de ativação elétrica e provavelmente a técnica de pedalada.

No estudo de Peveler, Pounders e Bishop (2007) o objetivo foi determinar se há diferença no desempenho dos ciclistas que adotam o método que recomenda posicionar o selim de modo a refletir um ângulo relativo do joelho entre 25 a $35^{\circ}$ $\left(0^{\circ}\right.$ extensão completa; para a prevenção de lesões) e o método que recomenda utilizar 109\% da altura do solo até a sínfise púbica (para o ótimo desempenho) para ajustar a altura do selim. Ciclistas treinados $(n=9)$ e não ciclistas $(n=18)$ foram avaliados por meio de um teste anaeróbico de produção de potência de $30 \mathrm{~s}$. Não houve nenhuma diferença significativa para o pico e para a média de potência produzida nos dois casos. $\mathrm{Na}$ situação com o ângulo do joelho em $25^{\circ}$ os ciclistas geraram uma potência significativamente mais alta comparado com o método de $109 \%$ da altura do solo até a sínfise púbica. Desta forma, é recomendado que a altura do selim seja regulada por meio da angulação do joelho entre 25 a $35^{\circ}$ para ciclistas destreinados, o que é relevante tanto para a prevenção de lesões quanto para 0 aumento do desempenho.

Peveler et al. (2005) compararam três métodos de ajuste da altura do selim existentes na literatura, a fim de verificar qual deles garantia que a angulação do joelho ficasse entre 25 e $35^{\circ}$ quando o pé encontra-se no ponto morto inferior do ciclo de pedalada. Os resultados sugeriram que o método que garante esta angulação é o proposto por Holmes (1994), no qual a altura do selim é determinada usando-se um ângulo de flexão do joelho entre 25 e $35^{\circ}$. Este método é recomendado por reduzir o risco de lesões de esforço repetitivo por excesso de treinamento.

Um dos efeitos relacionados em se desviar a altura do selim de seu posicionamento ótimo, além da possibilidade de levar à lesão, é que mais oxigênio passa a ser consumido pelo ciclista e mais trabalho é exigido, o que significa uma perda de eficiência (ALENCAR e MATIAS, 2009). Duc et al. (2008) investigaram a atividade muscular de 8 músculos do membro inferior e 4 músculos do membro superior durante o ciclismo. Dez ciclistas treinados pedalaram a $80 \%$ da sua carga aeróbica máxima em um cicloergômetro inclinado (4\%, $7 \%$ e $10 \%$ ) em duas posturas (sentado e de pé). A inclinação não provocou diferenças significativas na ativação muscular. Já a mudança de posição de pedalada da posição sentada para em pé, afetou grandemente a intensidade e o tempo de ativação EMG de todos os músculos, inclusive os que cruzam a articulação do tornozelo (gastrocnêmio medial, sóleo e tibial anterior), mas principalmente os dos braços e do tronco. $\mathrm{Na}$ posição sentada, a mudança do posicionamento das mãos no guidom (descanso e inclinado) teve pequeno efeito sobre a intensidade e o tempo de atividade EMG. Os principais efeitos da mudança de agarre no guidom da posição inclinada para a posição de descanso, sentado no selim, foi o aumento da atividade elétrica dos músculos dos braços. A atividade global dos músculos dos braços e do tronco foram superiores quando as mãos foram posicionadas na parte superior do guidom. O pico de atividade EMG do reto femoral foi menor nesta última posição. Estas mudanças podem ser explicadas pela alteração da posição dos ciclistas. Quando as mãos foram posicionadas na parte inferior do guidom, o centro de massa do corpo foi mais para trás e o tronco estava mais flexionado. Entretanto, os ciclistas apresentaram maior atividade dos músculos bíceps e tríceps braquial na posição de descanso porque o peso suportado pelos braços era maior. A coordenação entre os músculos antagonistas também foi alterada pela mudança da postura de pedalada. As mudanças estavam fortemente relacionadas com o aumento do pico de força aplicada ao pedal e a supressão do suporte do selim.

Estudar os efeitos de gênero, fatores antropométricos, potência, e posição das mãos no guidom sobre o movimento da pelve ao longo de um ciclo de pedalada foi objetivo do estudo de Sauer et al. (2007). Foram avaliados ciclistas experientes (12 homens e 14 mulheres). Cada sujeito foi avaliado em uma bicicleta estacionária em três produções de potência $(100,150$ e 200 $W)$, com as mãos na posição de descanso e aerodinâmica. A cinemática de uma tríade de marcadores, situados em marcos pélvicos posteriores, foi usada para caracterizar 0 movimento tridimensional. As excursões angulares maiores foram observadas com a pélvis rodando internamente $\left(\sim 3^{\circ}\right)$ e lateralmente $\left(\sim 2^{\circ}\right)$ para a posição aerodinâmica. A posição aerodinâmica causou no quadril inclinação nas direções anterior e inferior. Comparado com 
homens, as mulheres exibiram maior média de rotação pélvica anterior na posição aerodinâmica (homens: $21 \pm 3^{\circ}$; mulheres: $24 \pm 4^{\circ}$ ). A média do movimento pélvico anterior foi correlacionada negativamente com a flexibilidade lombar entre os homens, sugerindo que isto pode ser um importante fator a ser considerado ao se ajustar a bicicleta. Os autores concluíram que o movimento pélvico é esperado durante o ciclismo, e deveria ser considerado quando se estabelecem critérios para os procedimentos de ajuste da bicicleta.

Para o posicionamento da parte superior do corpo, os ciclistas geralmente ajustam seu alcance de acordo com seu conforto, de acordo com o nível de condicionamento dos músculos dorsais e a distância a ser percorrida (ASPLUND, WEBB e BARKDULL, 2005). Na categoria mountain bike, a posição superior do corpo é, na maior parte dos casos, próximo a vertical, já na categoria estrada, a posição mais inclinada funciona para diminuir a altura do centro de gravidade e distribuir melhor o peso entre a roda dianteira e traseira. Um tronco maior, braços mais longos, sobretudo o antebraço, em posição horizontal quando se agarra o guidom pela parte inferior, conduzirão a aumentar a distância selimguidom, o que logo se repercutirá também no comprimento do quadro e da mesa do guidom (MESTDAGH, 1998). O ângulo de inclinação do tronco é um fator importante por alterar as propriedades e recrutamento muscular durante a pedalada (SAVELBERG, VAN DE PORT e WILLEMS, 2003; DOREL, COUTURIER e HUG, 2007; DIEFENTHAELER et al., 2008a). Este ângulo de inclinação que caracteriza muitas posturas competitivas ainda causa posturas biomecânicas incorretas que geram diferentes padrões de sustentação sobre a bicicleta, por vezes requerendo o reforço de musculaturas específicas a fim de minimizar e/ou evitar futuras lesões/dores na coluna vertebral, como, por exemplo, desgastes de discos intervertebrais que podem causar hérnia de disco lombar (HENNEMANN e SCHUMACHER, 1994), vindo a afetar o desempenho do ciclista (SAVELBERG, VAN DE PORT, WILLEMS, 2003).

Analisar os efeitos de diferentes tipos de posicionamento no guidom sobre variáveis ventilatórias e metabólicas foi objetivo de estudo de Grappe et al. (1998). Foram utilizadas as posições vertical (tronco na vertical), inclinada (usada em provas de velocidade, no qual o atleta segura 0 guidom na porção inferior) e a aerodinâmica (usada em provas contra-relógio, a fim de diminuir a resistência do ar). Nove ciclistas competitivos foram avaliados em um cicloergômetro nas três condições, pedalando 10 minutos a $70 \%$ do $\mathrm{VO}_{2}$ max. As variáveis ventilatórias (ventilação, índice de troca respiratória, média inspiratória e percepção de esforço) foram significativamente maiores na posição inclinada do que na posição vertical. $\mathrm{VO}_{2}$, volume relativo, produção de gás carbônico, taxa respiratória, ciclo de débito inspiratório, freqüência cardíaca e pH permaneceram inalterados entre todas as posições analisadas. Estes resultados mostram que as maiores mudanças nas variáveis ventilatórias e metabólicas ocorreram na posição inclinada. A posição aerodinâmica é a posição mais satisfatória quando o arrasto aerodinâmico é predominante.

Ashe et al. (2003) compararam variáveis cardiovasculares e respiratórias de homens fisicamente ativos, assumindo-se duas posições no guidom (posição mais elevada e posição aerodinâmica). Os resultados demonstram que na posição com tronco mais elevado, foi observado maior consumo de oxigênio, ventilação, batimento cardíaco e carga máxima de trabalho, o que mostra o efeito de simples ajustes da bicicleta para ciclistas recreacionais.

Savelberg, Van de Port e Willems (2003) estudaram os efeitos da posição do tronco de ciclistas sobre o padrão de recrutamento muscular. Mudanças no posicionamento do tronco, para frente ou para trás, afetaram a cinemática do tornozelo e do quadril, bem como a orientação da coxa. Um padrão semelhante foi encontrado para a atividade elétrica muscular. Tanto para os músculos que cruzam a articulação do quadril quanto os que cruzam a articulação do tornozelo foram encontradas alterações no período de ativação e na amplitude do sinal eletromiográfico.

Dorel, Couturier e Hug $(2007)$ avaliaram
diferentes posicionamentos de triatletas
experientes sobre a bicicleta para verificar se este
fator poderia modificar a coordenação dos
membros inferiores durante a pedalada e,
conseqüentemente, influenciar a produção de
força no pedal. Os dados demonstraram um
aumento significativo no sinal eletromiográfico dos
músculos glúteo máximo e vasto lateral, e
diminuição da atividade do reto femoral na
posição aerodinâmica. As mudanças
eletromiográficas estão relacionadas com a
direção de aplicação da força na posição
aerodinâmica (altos picos de força positiva na
fase de propulsão, baixos picos de força negativa
na fase de recuperação, e ocorrência posterior
destes picos ao longo do ciclo do pé-de-vela, ou
seja, o ciclista aplica melhor a sua força e,
conseqüentemente, melhora seu desempenho)
comparando-se com as posições mais elevadas.


Segundo Burke e Pruitt (2003), uma boa bicicleta deve ter conforto e minimizar o potencial para lesões, para que dessa forma maximize 0 desempenho. Estes autores sugerem que o mais importante ângulo na bicicleta é a inclinação do tubo do selim (Figura 1). A inclinação ideal do tubo do selim requer que a face anterior da patela esteja sobre o eixo do pedal quando o pé-de-vela está na horizontal $\left(90^{\circ}\right)$. A inclinação do tubo do selim é geralmente projetada para satisfazer isso. Geralmente este ângulo fica entre $72^{\circ}$ e $74^{\circ}$, e os ajustes necessários são feitos com 0 posicionamento à frente e atrás do selim. A inclinação do tubo do selim está relacionada ao tamanho do fêmur. Adicionalmente, um ângulo relativo do joelho com extensão máxima entre $150^{\circ}$ e $155^{\circ}$ permitirá adequada descompressão na articulação do joelho prevenindo lesões na porção anterior da articulação patelofemoral (pela descompressão promovida) e evita o ponto morto inferior da descida do pedal. A posição neutra do joelho é determinada com um fio de prumo, para verificar a posição do joelho com o centro do pedal (MARTINS et al., 2007, Figura 3). Com a patela alinhada ao centro do pedal (pé-de-vela na horizontal - 90ㅇ) o joelho é considerado em posição neutra. Porém, de acordo com a especialidade de cada atleta se percebem preferências, como por exemplo, triatletas, que preferem uma posição onde o joelho estará um pouco mais a frente, resultado de uma posição também um pouco mais a frente sobre o selim.

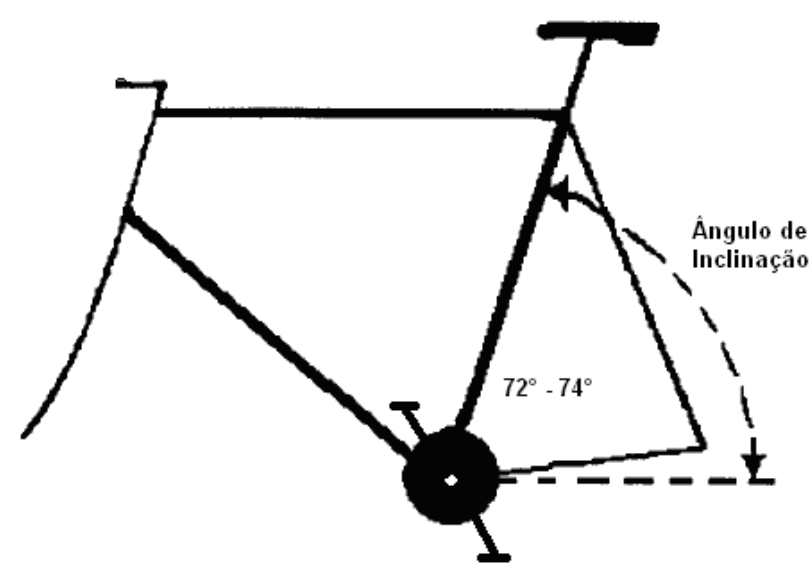

Figura 1. Representação esquemática do ângulo de inclinação do tubo do selim.

Salai et al. (1999) objetivaram avaliar as possíveis causas da dor lombar em ciclistas e sugeriram uma solução para o ajuste apropriado da bicicleta. Foram avaliados ciclistas em três tipos de bicicletas. A angulação pélvica e lombar foi mensurada assumindo-se posições diferentes sobre a bicicleta, e relatando os vetores de força atuando no períneo. A hiperextensão lombopélvica resultou em um aumento nas forças tensivas no períneo. Estas forças podem ser facilmente reduzidas com o apropriado ajuste da angulação do selim. Os achados da análise biomecânica, quando aplicados em um grupo de ciclistas, mostraram que após os ajustes adequados da angulação do selim, a maioria dos ciclistas $(>70 \%)$ apresentou diminuição na incidência e na magnitude das dores lombares. Os autores sugerem que um dos principais fatores relacionados à dor lombar no ciclismo é dependente do ajuste do selim. Por fim, a posição do pé e a liberdade no taco da sapatilha que mantém o pé conectado ao pedal devem ser analisadas individualmente para cada ciclista. A posição do engate na sapatilha é influenciada por muitas questões anatômicas, como a rotação da tíbia, pronação e alinhamento varo ou valgo dos joelhos (ALENCAR e MATIAS, 2009). A liberdade de movimento no pedal pode aliviar traumas e prover os ajustes necessários, devendo ser considerado como interligado com os demais ajustes, principalmente 0 do selim (MESTDAGH, 1998; BURKE e PRUITT, 2003).

O tamanho e o ajuste correto da geometria da bicicleta influenciam fortemente a transferência de potência da pedalada, a probabilidade de contrair lesões, o atrito aerodinâmico ou, mais diretamente, a sensação de conforto na bicicleta (MESTDAGH, 1998), principalmente por serem fatores que afetam aspectos da mecânica muscular, como as relações força-comprimento (HERZOG et al., 1991).

Bressel e Larson (2003) verificaram os efeitos de diferentes modelos de selins sobre os ângulos de tronco e pelve e conforto de ciclistas do sexo feminino. Foram avaliadas 20 ciclistas que pedalaram em uma bicicleta estacionária com as mãos posicionadas na parte superior e inferior do guidom e com a utilização de três modelos de selim (plano, parcialmente vazado e vazado). $O$ ângulo pélvico foi mensurado com um inclinômetro e o ângulo do tronco foi quantificado através de digitalização de imagens de vídeo. $O$ nível de conforto foi mensurado subjetivamente através de um ranqueamento do mais para o menos confortável. Seus principais resultados mostraram que a inclinação pélvica foi aumentada em $8 \%$ com a utilização do selim parcialmente vazado e em $16 \%$ com a utilização do selim vazado em relação ao selim plano. Os ângulos de flexão do tronco foram maiores com a utilização do selim vazado em comparação com os outros modelos. As participantes apresentaram um aumento de $77 \%$ de anteversão pélvica e de $11 \%$ de flexão do tronco quando utilizaram a posição 
aerodinâmica em relação a posição mais elevada no guidom. Um total de $55 \%$ dos sujeitos classificaram o selim parcialmente vazado como o mais confortável, e $30 \%$ classificaram o selim plano como o mais confortável. Estes dados indicam que os modelos de selim parcialmente vazado e vazado aumentam a inclinação pélvica anteriormente e que o selim vazado pode aumentar o ângulo de flexão do troco em algumas condições durante 0 ciclismo. Um selim parcialmente vazado pode ser mais confortável que os modelos plano e vazado.

\section{Conclusão}

Comumente as bicicletas nacionais vendidas para a população em geral apresentam apenas uma opção para o tamanho de quadro. Sendo que o tamanho do quadro é a medida da bicicleta que mais influencia todo o resto da geometria, sua limitação torna a bicicleta inviável de ser adaptada ao usuário. $O$ ideal seria o oferecimento de diversos modelos de acordo com a atividade que o usuário deseja, sendo disponibilizadas várias dimensões a fim de atender características específicas da população, como já ocorre em outros países. Em uma visão mais requintada, o ideal seria a confecção de bicicletas por encomenda. No entanto, essa estratégia envolve custos elevados e que muitas vezes não é o objetivo do praticante recreacional, o que fortalece a necessidade do uso de estratégias de avaliação.

Os estudos discutidos mostraram que o ajuste da bicicleta afeta não somente o desempenho, mas também o conforto e a saúde do praticante. Portanto, o ajuste adequado é importante na prevenção de lesões e maximização do prazer na prática esportiva. Os estudos também denotam a existência de várias propostas cientificamente validadas para ajustar corretamente o ciclista. Cada atleta deve ser individualmente ajustado, e este tipo de avaliação precisa ser popularizada entre os praticantes. As correções devem ser aplicadas uma de cada vez para que grandes alterações não sejam lesivas e nem causem desconforto no praticante.

\section{Referências}

ABRACICLO. Disponível em: http://www.abraciclo.com.br/. Acesso: 20 mai. 2008.

ALENCAR, T. A. M.; MATIAS, K. F. S. Bike fit e sua importância no ciclismo. Revista Movimenta, v. 2, n. 2, p. 59-64, 2009. Disponível em: http://www.nee.ueg.br/seer/index.php/movimenta/ article/viewFile/218/204. Acesso em: 27 out. 2009.
ASHE, M. C.; SCROOP, G. C.; FRISKEN, P. I.; AMERY, C. A.; WILKINS, M. A.; KHAN, K. M. Body position affects performance in untrained cyclists. British Journal of Sports Medicine, London, v. 37, n. 5, p. 441-444, 2003. http://dx.doi.org/10.1136/bjsm.37.5.441.

ASPLUND, C.; WEBB, C.; BARKDULL, T. Neck and back pain in bicycling. Current Sports Medicine Reports, v. 5, p. 271-274, 2005. http://dx.doi.org/CSMR.0000306221.25551.69.

BRESSEL, E.; LARSON, B. J. Bicycle seat designs and their effect on pelve angle, trunk angle and comfort. Medicine and Science in Sports and Exercise, v. 35, n. 2, p. 327-332, 2003.

http://dx.doi.org/?10.1249/01.MSS.0000048830.22 $\underline{964.7 \mathrm{c} .}$.

BURKE, E. R. High-Tech Cycling: the faster rider. Colorado Springs: Human Kinetics, [1996].

BURKE, E. R. Physiology of cycling. In: GARRRET, W. E.; KIRKENDALL, D. T. (Org.) Exercise and Sport Science. Lippincott Williams \& Wilkins. Philadelphia, 2000. p. 759-770.

BURKE, E. R.; PRUITT, A. L. Body positioning for cycling. In: BURKE, E. R. (Org.) High-Tech Cycling. 2 ed. Champaign: Ed. Human Kinetics, 2003. p. 69-92.

CARMO, J. C. Biomecânica aplicada ao ciclismo. In: CONGRESSO BRASILEIRO DE BIOMECÂNICA, 9, 2001, Gramado. Anais... Gramado: Sociedade Brasileira de Biomecânica, 2001. p. $42-47$.

CHRISTIAANS, H. H. C. M.; BREMNER, A. Comfort on bicycles and the validity of a commercial bicycle fitting system. Applied Ergonomics, Kidlington, v. 29, n. 3, p. 201-211, 1998. http://dx.doi.org/10.1016/S00036870(97)00052-5.

DIEFENTHAELER, F.; BINI, R. R.; KAROLCZAK, A. P. B.; CARPES, F. P. Ativação muscular durante a pedalada em diferentes posições do selim. Revista Brasileira de Cineantropometria e Desempenho Humano, Florianópolis, v. 10, n. 2, p.161-169, 2008a. Disponível em: http://www.rbcdh.ufsc.br/MostraEdicao.do?edicao =36. Acesso em: 15 out. 2009.

DIEFENTHAELER, F.; BINI, R. R.; NABINGER, E.; LAITANO, O.; CARPES, F. P.; MOTA, C. B.; GUIMARÃES, A. C. S. Proposta Metodológica para a Avaliação da Técnica da Pedalada de Ciclistas: Estudo de Caso. Revista Brasileira de Medicina do Esporte, São Paulo, v.14 n. 2, p.145-148, 2008b. Disponível em: http://dx.doi.org/0.1590/S151786922008000200014. 
DONKERS, P. C. M.; TOUSSAINT, H. M.; MOLENBROEK, J. F. M.; STEENBEKKERS, L. P. A. Recommendations for the assessment and design of young children's bicycles on the basis of anthropometric data. Applied Ergonomics, Kidlington, v. 24, n. 2, p. 109-118, 1993. http://dx.doi.org/10.1016/0003-6870(93)90082-K.

DOREL, S.; COUTURIER, A.; HUG, F. Influence of different racing positions on mechanical and electromyographic patterns during pedalling. Scandinavian Journal of Medicine \& Science in Sports, Copenhagen, v. 19, n. 1, p. 44-54, 2007. http://dx.doi.org/10.1111/j.1600$\underline{0838.2007 .00765 . x}$.

\section{DUC, S.; BERTUCCI, W.; PERNIN, J.N.;} GRAPPE, F. Muscular activity during uphill cycling: Effect of slope, posture, hand grip position and constrained bicycle lateral sways. Journal of Electromyography and Kinesiology, v. 18, p. 116-127, 2008.

http://dx.doi.org/10.1016/j.jelekin.2006.09.007.

GRAPPE, F.; CANDAU, R.; BUSSO, T.; ROUILLON, J. D. Effect of cycling position on ventilatory and metabolics variables. International Journal of Sports Medicine, v. 19, n. 5, p. 336341, 1998. Disponível em: http://www.thiemeconnect.de/ejournals/toc/sportsmed. Acesso em: 02 out. 2009 .

HENNEMANN, S. A.; SCHUMACHER, W. Hérnia de disco lombar: revisão de conceitos atuais. Revista Brasileira de Ortopedia, v. 29, n. 3, p. 115-126, 1994. Disponível em: http://www.rbo.org.br/. Acesso em: 26 jul. 2007.

HERZOG, W.; GUIMARÃES, A. C.; ANTON, M. G.; CARTER-ERDMAN. K. A. Moment-length relations of rectus femoris muscles of speed skaters/cyclists and runners. Medicine and Science in Sports and Exercise, Baltimore, v. 23, n. 11, p. 1289-1296, 1991. Disponível em: http://www.ncbi.nlm.nih.gov/pubmed/1766346. Acesso em: 23 ago. 2003.

LAIOS, L.; GIANNATSIS, J. Ergonomic evaluation and redesign of children bicycles based on anthropometric data, Applied Ergonomics, p. 18, 2009.

http://dx.doi.org/10.1016/j.apergo.2009.09.006.

MARTINS, E. A.; DAGNESE, F.; KLEINPAUL, J. F.; CARPES, F. P.; MOTA, C. B. Avaliação do posicionamento corporal no ciclismo competitivo e recreacional. Revista Brasileira de Cineantropometria e Desempenho Humano, Florianópolis, v. 9, n. 2, p.183 -188, 2007. Disponível em: http://www.rbcdh.ufsc.br/MostraEdicao.do?edicao $=30$. Acesso em: 12 out. 2009 .

MESTDAGH, K. V. Personal perspective: in search of an optimum cycling posture. Applied
Ergonomics, Kidlington, v. 29, n. 5, p. 325-334, 1998. http://dx.doi.org/10.1016/S00036870(97)00080-X.

PEQUINI, M. P. A evolução tecnológica da bicicleta e suas implicações ergonômicas para a máquina humana: problemas da coluna vertebral $\mathrm{x}$ bicicletas dos tipos Speed $\mathrm{e}$ Mountain Bike. 2000, 300f. Dissertação (Programa de Pós-Graduação em Arquitetura e Urbanismo). Universidade de São Paulo, São Paulo (SP), 2000. Disponível em: http://www.portaldeconhecimentos.org.br/index.ph p/por/content/view/full/10332. Acesso em: 20 jun. 2008.

PEVELER, W. W.; BISHOP, P. A.; SMITH, J.; RICHARDSON, M.; WHITEHORN, E. Comparing methods for setting saddle height in trained cyclists. Journal of Exercise Physiology online, v. 8, n. 1, p. 51-55, 2005. Disponível em: http://www.asep.org/files/PevelerSaddle.pdf. Acesso em: 20 out. 2009.

PEVELER, W. W.; POUNDERS, J. D.; BISHOP, P. A. Effects of saddle height on anaerobic power production in cycling. Journal of Strength and Conditioning Research / National Strength \& Conditioning Association, Colorado, v. 21, n. 4, p. 1023-1027, 2007. Disponível em: http://dialnet.unirioja.es/servlet/articulo?codigo $=24$ 99782. Acesso em: 23 out. 2009.

ROWE, T.; HULL, M. L.; WANG, E. L. A Pedal Dynamometer for Off-Road Bicycling. Journal of Biomechanical Engineering, Pittsburg, v. 120, n. 1, p.160-164, 1998. http://dx.doi.org/10.1115/1.2834297.

SALAI, M.; BROSH, T.; BLANKSTEIN, A.; ORAN, A.; CHECHIK, A. Effect of changing the saddle angle in the incidence of low back pain in recreational bicyclists. British Journal of Sports Medicine, London, v. 33, n. 1, p. 398-400, 1999. Disponível em: http://journals.bmj.com. Acesso em: 23 out. 2009.

SAUER, J. L.; POTTER, J. J.; WEISSHAAR, C. L.; PLOEG, H. L.; THELEN, D. G. Influence of Gender, Power, and Hand Position on Pelvic Motion during Seated Cycling. Medicine and Science in Sports and Exercise, Baltimore, v. 39, n. 12, p. 2204-2211, 2007.

http://dx.doi.org/10.1249/mss.0b013e3181568b66.

SAVELBERG, H. H. C. M.; VAN de PORT, I. G. L.; WILLEMS, P. J. B. Body Configuration in Cycling Affects Muscle Recruitment and Movement Pattern. Journal of Applied Biomechanics, Delaware, v. 19, n. 4, p. 310-324, 2003. Disponível em:

http://journals.humankinetics.com/jab-backissues/JABVolume19Issue4November. Acesso em: 23 out. 2009. 
SILBERMAN, M. R.; WEBNER, D.; COLLINA, S.; SHIPLE, B. J. Road Bicycle Fit. Clinical Journal of Sport Medicine : Official Journal of the Canadian Academy of Sport Medicine,

Baltimore, v. 15, n. 4, p. 271-276, 2005.

WELBERGEN, E.; CLIJSEN, L. P. V. M. The influence of body position on maximal performance in cycling. European Journal of Applied Physiology, Heidelberg, v. 61, n. 1-2, p. 138-142, 1990.

http://dx.doi.org/10.1007/BF00236708.

Agradecimentos: JFK, FD e FPC agradecem ao apoio da Universidade Federal de Santa Maria e Universidade Federal do Rio Grande do Sul aos projetos desenvolvidos na área de biomecânica do ciclismo, bem como aos integrantes do GEPEC pelo suporte aos projetos do grupo. Os protocolos de avaliação descritos nesse artigo são desenvolvidos pelo GEPEC e podem ser solicitados por qualquer pessoa, seja ciclista recreacional, seja competitivo. Os autores também agradecem a todos os autores e sujeitos envolvidos nos estudos avaliados, pois sem eles esse conhecimento não teria sido produzido. Este estudo não recebeu financiamento para ser realizado.

\section{Endereço:}

Julio Francisco Kleinpaul

R. João Agostinho Vieira, 748. Ribeirão da llha.

Florianópolis SC Brasil.

88040-000

E-mail: juliofk@gmail.com

Recebido em: 30 de novembro de 2009.

Aceito em: 4 de maio de 2010.

\section{cc) (i) (9)}

Motriz. Revista de Educação Física. UNESP, Rio Claro, SP, Brasil - elSSN: 1980-6574 - está licenciada sob Licença Creative Commons 